\title{
Rezensionen
}

\section{Review Essay: Religion und Wirtschaft: Endlich wieder gemeinsam im Blick?}

DOI 10.1515/zfr-2014-0015

François Gauthier und Tuomas Martikainen, Hg., Religion in the Neoliberal Age. Political Economy and Modes of Governance, Ashgate AHRC/ESRC.

Religion and Society Series (Farnham, Burlington VT: Ashgate 2013), 248 S., ISBN 978-1-4094-4978-2, f 65,00 (= 2013a).

François Gauthier und Tuomas Martikainen, Hg., Religion and Consumer Culture. Brands, Consumers and Markets, Ashgate AHRC/ESRC. Religion and Society Series (Farnham, Burlington VT: Ashgate 2013), 250 S., ISBN 978-1-4094-4986-7, f 65,00 (= 2013b).

Jörg Stolz und Jean Claude Usunier, Hg., Religions as Brands. New Perspectives on the Marketization of Religion and Spirituality, Ashgate AHRC/ESRC. Religion and Society Series (Farnham, Burlington VT: Ashgate 2014), 256 S., ISBN 978-1-4094-6755-7, f 65,00.

Spätestens seit den 1980er Jahren ist der Neoliberalismus das sozio-ökonomische Rahmenwerk, innerhalb dessen Religion in all ihren Organisationsformen in den meisten Ländern steht und sich entwickelt. Daher ist dieser Rahmen kaum noch zu separieren von Religionskulturen und sollte in der Beschreibung von Religion berücksichtigen werden. Dieses Erfordernis resultiert auch daraus, dass der neoliberale Kapitalismus weitgehend zur kulturellen Ideologie geworden ist. Organisationen reagieren in diesem ökonomischen Einflussfeld nicht nur in eine Richtung. Mal tragen sie zur Verstärkung von Prozessen der Ökonomisierung bei, zum Beispiel wenn Erfolg im Versprechen finanziellen Heils naturalisiert wird, mal opponieren sie gegen diesen Trend, jedoch kaum auf ganzer Linie, sondern indem sie typischerweise einige neoliberale Elemente nach wie vor übernehmen. Solche Elemente können zum Beispiel das outsourcing von einzelnen Dienstleistungen sein (psychologische Teamsupervision oder Catering von kirchlichen Einrichtungen) oder das Abwälzen hoher Risiken auf den Einzelnen (Kündigung des Arbeitsverhältnisses in der katholischen Kirche bei Verstoß gegen religiöse Lehre wie bei

Anne Koch: München, Email: anne.koch@evtheol.uni-muenchen.de 
geschieden Wiederverheirateten oder dem Eingehen einer eingetragenen Partnerschaft). Neoliberalismus wird mit weiteren Elementen verbunden wie mit flexibleren Produktionsweisen, segmentierten Märkten, der Verdichtung von Raumzeit, der globalen Vernetzung und Ausbreitung von Arbeitsprozessen, Wettbewerb, der Privatisierung auch von Wohlfahrt und einer hohen Selbstverantwortung des Einzelnen für Vorsorge und Arbeitskraft. Unter diesen Bedingungen sei eine neue „religio-ethno-economic practice“ als integraler Bestandteil des neoliberalen Kapitalismus entstanden (Gauthier und Martikainen 2013a). Das macht nach Auffassung von François Gauthier und Tuomas Martikainen die religionswissenschaftliche Relevanz dieser Untersuchungen zu Religion und Wirtschaft aus. Dem kann man sich nur anschließen. Es ist also höchste Zeit sich intensiver diesen Verstrickungen zuzuwenden.

Der Literatur- und Kulturwissenschaftler Joseph Vogl hat jüngst nachdrücklich die zutiefst normative Ideologie des Kapitalismus über die historische Rekonstruktion ihres Narrativs bloßgestellt (2010). Im Zentrum steht ein autopoietischer Markt, der seit Adam Smith nicht nur sich, sondern zugleich Gesellschaft ausbalanciert. Diese jahrhundertealte „Oikodizee“ habe nur ein Problem: nach der Serie globaler Finanzkrisen würde kaum noch jemand dem „Gespenst des Kapitals“ glauben. Jedoch ist damit noch nicht die ganze religionsökonomisch erforderliche Aufarbeitung des neoliberalen Kapitalismus vollbracht. Vogls Band liest sich großenteils auch deshalb so prägnant, weil Wirtschaftsgeschehen eindrucksvoll vereinfacht wird.

Nach den in der deutschsprachigen Religionswissenschaft gänzlich verpufften, obwohl bislang einzigen Monographien zu Religion und Wirtschaft, den wirtschaftswissenschaftlichen Dissertationen von Christian Eilinghoff (deutlich neo-klassisch) und Ron Brinitzer (neo-institutionenökonomisch), ist nun endlich Bewegung in das Themengebiet gekommen. Markus Heros Dissertation (2011) ist eine sehr aufschlussreiche institutionensoziologische Analyse des Dienstleitungsmarktes alternativer Heilweisen und neureligiöser Angebote. Mit dem monströsen The Oxford Handbook of the Economics of Religion, herausgegeben von der Harvard-Philosophin Rachel McCleary, ist nun scheinbar auch ein Handbuch auf dem Markt, das in „die“ Religionsökonomie einführt. Doch sind die Beiträge fast ausnahmslos wie das ganze sehr interessante Werk von McCleary und ihrem Mann Robert Barro (ebenfalls Harvard, ökonomische Wachstumstheorie) im Paradigma der Neoklassik und Rationalwahl verfasst und verstehen unter Religion vor allem religiöse Firmen und nutzenmaximierende Einzelne. So erstaunt nicht, dass McClearys Vergleich von Errettungs- und Verdammungsszenarien als ökonomische Anreizsysteme über mehrere „Weltreligionen“ hinweg religionswissenschaftlich eher abenteuerlich ist. Desgleichen gilt für Brinitzers Religionsbegriff - was der Qualität der neo-institutionenökonomischen Analyse nicht 
wirklich schadet (es sind eben statt informeller Religion und kultureller Muster bei ihm nur klassische Akteure wie Kirchgänger und religiöse Organisationen im Blick, doch die nach allen Regeln der Kunst). Wie in vielen ökonomischen Arbeiten zu Religion sind kulturwissenschaftliche Erkenntnisse kaum rezipiert: Das Tabukonzept etwa ist bei Brinitzer nicht in seiner fremdheitskonstruktiven Herkunft problematisiert und begriffsgeschichtlich diskutiert. Das gleiche gilt für die Universalisierung des Heiligen: „Neben Tabuvorstellungen ist der Begriff der Heiligkeit allen Religionen inhärent“ $(2003,67) .{ }^{1}$ Brinitzers Institutionalismus kommt am stärksten in den Theoriestücken zu geteilten mentalen Modellen, zur Historizität von Institutionen mit ihren Pfadabhängigkeiten und zu Transaktionskosten zur Geltung. Im Streit darüber, ob die Neue Institutionenökonomik noch stark in Kontinuität zur Neoklassik der Rationalwahl stehe oder eine neue Theorie sei, sind die genannten Arbeiten gewiss eher in Kontinuität mit der Neoklassik zu sehen.

Vor diesem Hintergrund bisheriger Monographien sind die drei jüngsten Sammelbände bei Ashgate ein großer Schritt. Sie machen insbesondere religionssoziologische, wenn auch immer noch nicht einschlägige wirtschaftssoziologische Erkenntnisse für in den meisten Beispielen gegenwärtige Religion im ökonomischen Kontext fruchtbar.

Gauthiers und Martikainens Band „Religion in the Neoliberal Age“ ist aus einer ISSR-Konferenz 2011 in Aix-en-Provence hervorgegangen. Für Gauthier (damals noch University of Montréal, jetzt Fribourg/CH) und Martikainen (University of Helsinki) ist der Neoliberalismus ein sehr viel relevanteres und sträflich vernachlässigtes Paradigma des religiösen Feldes als etwa der beliebte Postsäkularismus. Programmatisch sprechen sie von der Notwendigkeit einer Wende in der religionswissenschaftlichen Theoriebildung, die trotz ihrer ,inter-, cross- and trans-disciplinarity“ $(2013,3)$ kaum neue Konzepte erarbeitet habe jenseits vom Clash der Kulturen, Multikulturalismus und der Säkularisierungsdebatte. In einem ersten Survey bisheriger Literatur zu Religion und Wirtschaft bestimmen sie vier thematische Felder, auf denen bisherige Untersuchungen sich bewegen. Da sind zunächst 1. ideologisch-kulturpessimistische Arbeiten; 2. Arbeiten, die den Einfluss von Religion und Wirtschaft eher positiv bestimmen; 3. Literatur zu Wohlstandsreligion; 4. jüngste Literatur zu Religion im Rahmen neoliberaler politischer Ökonomie (2013, 5-10). Die Vertreter der ersten Gruppe bezeichnen

\footnotetext{
1 Seine Auskunftsquellen in Sachen Religionstheorie sind lediglich H.-J. Schoeps' substanzieller Religionsbegriff aus Religionen - Wesen und Geschichte (1963) und Pascal Boyers Religion explained. Auch transzendent und transzendental werden häufig verwechselt (2003, 59; 160 Anm. 66) wie bei dem neoklassischen Ökonom Dieter Schmidtchen in seinem neoklassischen Beitrag im damaligen Sonderheft zur Religionsökonomie in dieser Zeitschrift im Jahr 2000.
} 
Gauthier und Martikainen auch als „(neo-)marxistische“ Tradition (denn ökonomische Faktoren „entfremden“) und „nostalgisch“ aufgrund eines essentialistischen Verständnisses einer von Wirtschaft unbefleckten Religion, deren Verschwinden bedauert werde. Es ist fragwürdig, ob sie mit dieser Charakterisierung alle relevanten kulturkritischen Arbeiten erfassen können, da die Entfremdung des Menschen von seiner Arbeit nicht immer der zentrale Kritikpunkt ist. Zum Teil ist es auch die angebliche Verfälschung einer kulturellen Tradition in der Rezeption und der Missbrauch von Spiritualität von Unternehmen etwa zur Formulierung ihrer Ziele, die kritisiert werden (zum Beispiel bei Carrette und King 2005). In die zweite Gruppe von Literatur fallen P.L. Berger (denominationaler US-Wettbewerbsmarkt), W.C. Roof (,spiritual marketplace“), Rational Choice-Soziologen und J. Stolz (soziologische Rationalwahl). Diese seien ideologisch, da sie begeistert von kompetitiven Märkten ausgingen und damit gänzlich in der neoliberalen Ideologie beheimatet wären. Warum Wohlstandsreligion als eines von vielen wirtschaftsethnologischen Konzepten eine eigene Gruppe 3 ausmachen soll, leuchtet nicht ganz ein. Ebenso hätten okkulte Ökonomien, Ritualökonomien, die Kommodifizierung von ethnischer oder religiöser Identität und einige weitere relevante Themen ausgesucht werden können, die als Wiederverzauberung „unter Druck des Kapitalismus“ Erwähnung finden (2013a, 8).

Die Beiträge des Bandes zählen sich alle zum Punkt 4: „Religion im Rahmen neoliberaler politischer Ökonomie“. Neoliberale Ideologie wird über zwei ihrer Kennzeichnen erläutert: über den Konsumismus als Ethos unserer Gesellschaft und über Management, verstanden als Einzug von rationalen Verfahren der Effizienzsteigerung in soziale Beziehungen bis hin zum Selbstmanagement. Die immens wichtige Funktion dieser beiden Entwicklungen in den Nachkriegsgesellschaften ist an vielen Orten erforscht worden. Man denke in Bezug auf Unternehmenskommunikation und -führung an Eva Illouz' hervorragende historische Rekonstruktion des emotionalen Kapitalismus schon seit Beginn des 20. Jahrhunderts. Gauthier/Martikainen führen den weniger spezifischen Charles Taylor für diesen Punkt an, da sie eher an den modernen Individualismus denken, dem sich in Konsumkulturen ungeahnte Formen von Authentizität eröffneten. Weitere wichtige Gestaltungsfaktoren im Neoliberalismus sind die neuen Medien und transnationale Institutionen an deregulierten Finanzmärkten. Folgerichtig machen die Themenfelder „Religion und Governance“ (also die dazugehörige Regulationsform) und einzelne „religiöse Organisationen unter neoliberalen Bedingungen“ die beiden Teile des Bandes aus.

So wichtig das Konzept des Neoliberalismus ist und so fundiert und plausibilisierend seine historische Genese einleitend dargestellt wird, so allgemein ist diese Bestimmung des Rahmens unter religionsökonomischer Beurteilung. Hier hätte man sich einführende Worte zur spezifisch finanzmarktlichen Form der- 
zeitiger Kapitalismen gewünscht und eine Selbstverordnung, etwa ob man sich methodisch der Neo-Institutionenökonomik anzuschließen gedenkt, wie man sich zur new economic sociology verhält, schließlich auch eine genauere Differenzierung der „postfordistischen“ Arbeitswelt auf der Grundlage gegenwärtiger wirtschaftssoziologischer und wirtschaftswissenschaftlicher Diskussion.

Die Beiträge des Bandes sind durchgehend anspruchsvoll, argumentativ stark und an interessantem religionsgeschichtlichen Material entfaltet. Die von Gauthier und Martikainen angeführte neue „religio-ethno-economic practice“ findet in den Beiträgen daher eine exzellente Demonstration und Entfaltung. Ohne auf jeden einzelnen eingehen zu können, sei Joanildo A. Buritys überaus aufschlussreicher Vergleich angeführt von ,unternehmerischer Spiritualität“ mit dem „ökumenischen Alterglobalismus“ als zweier unterschiedlicher religiöser Reaktionen auf den globalen Neoliberalismus. Ein mustergültiges Anwenden von Foucaults Gouvernementalität auf Religion findet sich bei Breda Grey. Sie zeigt, wie die katholisch zivilgesellschaftliche Flüchtlingshilfe in Irland neoliberale Instrumente wie zum Beispiel eine „audit culture“ adaptiert, um ihre Ziele effizient $\mathrm{zu}$ erreichen. Beide Beiträge zeigen, wie neoliberale Tendenzen zur Privatisierung öffentlicher Leistungen und in der Folge $\mathrm{zu}$ joint ventures aus privatem, religiösem und staatlichem Sektor entstehen, um Effizienz zu steigern und im Wettbewerb standzuhalten, der selbst die Herstellung öffentlicher Güter erfasst hat. Einige Beiträge gehen auf die Bereitstellung von Wohlfahrt durch nordamerikanische (Jason Hackworth; David Ashley und Ryan Sandefer) und kanadische (Lori G. Beaman) religiöse Organisationen ein. Agnes Chong erläutert, wie die Verschärfung der Finanzierungsgesetze für Vereine in Australien als Reaktion auf den 11. September die ökonomische Basis vieler Moscheevereine stark verändert und beleuchtet damit die Auswirkungen des Rechts auf das institutionelle Feld. Jens Schlamelcher zeigt an protestantischen Citykirchen als niedrigschwelligem, undoktrinärem und sozial losem Angebot in Deutschland, wie in Seelsorge, Pflege und Verwaltung neo-liberale Verfahren greifen. Outsourcing, Kundenorientierung und ein steigender Bedarf an Kommunikationsorganisation rühren vor allem aus Finanzierungserfordernissen her und weniger aus freiem Entschluss. Entlarvend in Bezug auf unsere akademischen Interpretationen von religiösem Revival und neuem Fundamentalismus ist James V. Spickards Beitrag, der den Erfolg des Neoliberalismus-Paradigmas mit der Möglichkeit auf Reichtum für mächtige Länder erklärt und somit aus einer nicht eurozentrischen Perspektive beschreibt.

Im Neoliberalismus haben sich gesellschaftliche Strukturen und damit zusammenhängend Kommunikation und Regulation zwischen den beteiligten Akteuren grundlegend geändert. Das eher hierarchisch verfasste Staats-KirchenSystem wird bereits ergänzt durch eine multireligiöse Marktregulation mit Wett- 
bewerbsbedingungen und vielen genannten neoliberalen Elementen. Viele der Beiträge verfolgen diese veränderte Governance von Religion. Tuomas Martikainen führt am Beispiel Finnlands die dritte Form einer mehrschichtigen und plurifokalen Regulation über Netzwerke aus. Diese sind vor allem durch die Migrationssituation nach Auflösung des Ostblocks und neue Akteure im Bereich von Wohlfahrt, Migration und Flüchtlingshilfe etc. bedingt. Ähnlich verhält es sich in Estland (Ringo Ringvee). Sehr deutliche Thesen formulieren Rachel Chagnon und François Gauthier über den Einzug des Neoliberalismus in die juristische Verfahrensrationalität und das Rechtsverständnis Kanadas. Den Boden habe dafür eine Veränderung der moralischen Grundfesten bereitet im Zuge der Säkularisierung.

In ihrem zweiten Band „Religion in Consumer Society“ legen Martikainen und Gauthier den Schwerpunkt auf das Ethos des Konsumierens als Medium des modernen Individualismus anstatt auf institutionalisierte Religion und Fragen der Regulation (Governance) wie noch im ersten Band. Die beiden Teile des Bandes, „sich wandelnde Weltreligionen“ (I) und „kommodifizierte Spiritualitäten“ (II), machen nur Sinn vor der religionssoziologischen Fundamentalunterscheidung von formalen und informellen Organisationstypen. Die Bewertung der Folgen der Konsumgesellschaften auf Religion können auseinandergehen, wie Simon Speck in seinem sehr guten Beitrag zum konstitutiven Zusammenhang von verschränkter Moderne und kommodifizierter Religion ausführt: Während Zygmunt Baumann eine Trivialisierung von Religion beobachte, so fände für Ulrich Beck gerade eine Revitalisierung von Religion statt. Allerdings entspringe dieses Konzept einer kosmopolitischen Religiosität bei Beck einem Zirkelschluss: das schiere Faktum globalisierter Marktinteraktion lasse noch keine globale Gesellschaft entstehen, die als Prinzip einer universalen Menschheit daher auch nicht eine kosmopolitische Religiosität einführe. Christliche Kirchen reagierten auf die Ökonomisierung mit einem stärkeren Interaktionismus wie im Falle der schwedischen Lutherischen Kirche (Per Petterson) oder wie im Falle US-amerikanischer Megachurches, indem sie ein hochemotionales Produktpaket Protestantismus schnürten (Stephen Ellingson). Der Katholizismus erlebe eine erhöhte Nachfrage nach monastischem Lifestyle infolge des II. Vatikanums, die - so zeigt Stefania Palmisano für Klöster in Italien - eine konsumistische Form entwickelt habe, in der Wohlbefinden und Subjektivismus zentral seien. Mira Niculescus Beitrag thematisiert die massive Erweiterung der Produktpalette des amerikanischen Judentums mit Neo-Kabbalah als mystischem Angebot, seine pop-kulturelle Überarbeitung („Re-branding“) und die Aufnahme eines Potpourris „fernöstlicher“, neu-buddhistischer Diskurse.

Andrew Dawsons Beitrag, der den zweiten Teil über „kommodifizierte Spiritualität“ eröffnet, verankert das urbane professionelle soziale Milieu, in dem 
sich Alternativreligiosität weitgehend bewegt, im Modell der verwobenen Moderne. Er folgert daraus als Kennzeichen der Trägerschicht, dass sie als autonome Verbraucher meist am eigenen Körper sich verwirklichen und Heilung, Transformation usw. herstellen. Das spirituelle Repertoire an Handlungen und Inhalten ist dazu provisorisch, offen für viele Interpretationen, holistisch und expressiv und untersteht zum Teil ambivalenten Dynamiken, die Dawson pointiert ausführt und mit der starken These vertritt, sie weltweit in urbanen Zentren vorzufinden. Gauthier erschließt mit dem Begriff der Verzauberung die Ökonomisierung am Beispiel anti-kapitalistischer, informeller Religion: Das Burning Man Festival in der Wüste Nevadas im Geiste der säkularistischen Selbsttherapiekultur Esalens, des modernen Schamanismus (als Zusammenkunft von Stämmen) und des New Age führt für eine Dauer von einigen Tagen eine Tauschökonomie ein. Das Festival übt Konsumkritik mit einem verzaubernden Feuerritual als Höhepunkt. Konsumkritik sei gleichsam eine Ausnahme in einer Gesellschaft, in der Authentizität auf die Selbstaussage über Konsum angewiesen sei (auch Prosumption genannt aus Produktion und Verbrauch, engl. consumption). Rokpa International, eine Wohltätigkeitsorganisation in der Tradition des tibetischen Buddhismus, wird von John S. McKenzie in ihren aktiven Anpassungsstrategien an Konsumgesellschaften analysiert. Dazu gehören Tee-, Devotionalien- und Buchläden, Vorträge und Kurse in der Lehre gegen Gebühren, Therapien und Retreats in den Männer- und Frauenklöstern des Rokpa sowie Bemühungen um Kundenbindung. Diese Anpassung ermögliche auf der Nachfragerseite, kulturell-religiöse Bedürfnisse mit Konsumwünschen $\mathrm{zu}$ verbinden und auf Anbieterseite die religiöse Lehre zu finanzieren.

Lars Ahlin setzt an bei der bemerkenswerten Koinzidenz von angeblich kapitalismuskritischer New-Age-Missionierung und zugleich einer eindeutig neoliberal positionierten Propaganda in der größten schwedischen Tageszeitung in den 1980er Jahren. Das lässt ihn fragen, was die gemeinsamen Interessen von New Age und Neoliberalismus in jener Zeit waren. Er stößt dabei vor allem auf die Verheißung der Freiheit für Individuen, die im Kalten Krieg zwar nicht eine sozialistische, wohl aber eine humanistische, konsensorientierte kapitalistische Gesellschaft anstreben. Ebenfalls für Schweden geht Anne-Christine Hornborg der Ökonomisierung des Heilens im Coaching nach, wo die Grenze zur Therapie immer stärker verwischt. Am Beispiel der ausdrücklich mit „spirituell“ werbenden schwedischen Firma CoachCompanion zeigt sie die Transformation des gegenkulturellen New Age von Brandon Bays The Journey-Methode zum „Next“ Age auf, in dem Wohlstand klar mit Selbstverwirklichung verknüpft ist. Besonders relevant wird dieses Coaching in Schweden, da es von öffentlicher Hand für Lehrer, Schüler und Angestellte eingekauft wird. Für das westenglische Glastonbury beschreibt Marion Bowman einen ausdifferenzierten spirituellen Dienstleis- 
tungsmarkt. Den spirituellen Unternehmern ist die Synergie des multireligiösen Marktes sehr bewusst und wird positiv bewertet.

Jörg Stolz' und Jean Claude Usuniers „Religions as Brands“ geht auf eine Konferenz 2011 in Lausanne zurück. Jörg Stolz hat sich mit einigen Beiträgen in der Religionsökonomie in der Tradition der soziologischen Rationalwahl hervorgetan und von Weber ausgehend eine Taxonomie von Heilsgütern entwickelt (2008). Konsumverhalten und Marketingkommunikation in transkultureller Perspektive sind die Forschungsfelder des ebenfalls Lausanner Ökonomen Usunier. Marketing mit einem Fokus auf Markenbildungen ist zentrales Thema des Bandes. $^{2}$

Die vier Teile von „Religions as Brands“ werden eingeleitet von einem Forschungsüberblick einschlägiger Beiträge rund um Marke und Vermarktung von Religion durch Stolz/Usunier und Usuniers Beitrag darüber, wie die Produktklassifikation der Welthandelsorganisation „religiöse Dienstleistungen“, die seit 1995 ins Regelwerk aufgenommen ist, die Kommodifizierung von Religion vorantreibe. Wenig einleuchtend und methodologisch, aus einer ökonomischen Perspektive auch überflüssig ist Stolz/Usuniers Klassifikation von Organisationen aufsteigend zum unterschiedlichen Transzendenzbezug von Null-Religion/Spiritualität, säkularer und hybrider Religion/Spiritualität und Religion/Spiritualität. Denn wie und vor allem warum sollten „,sports yoga“, „hybrid yoga“ und „,spiritual yoga“ $(2014,12)$ zum Beispiel aussagekräftig voneinander abgrenzbar sein?

Teil II des Bandes untersucht unter dem sehr allgemeinen Titel „Branding and Marketing“ so unterschiedliche Dinge wie das Event Management (Olivier Favre), die lokale Adaption von Pop/Rockmusik in der geistlichen Musik der australischen Megachurch Hillsong und deren Produktion einer globalen Marke (Thomas Wagner - wie auch schon Marion Maddox 2013) oder die Marketingstrategien des spirituellen Kleinunternehmers (Markus Hero). Jason Dean untersucht den Slogan „Islam du juste milieu“ (nach Sure 2) in Frankreich, mit dem einer der Wettbewerber muslimischer Organisationen in der Kandidatur um den Vorsitz im französischen Muslimrat erfolgreich war. Allerdings sei dieser Slogan nicht als Marke sinnvoll zu erläutern, da Marken der Produktdifferenzierung an einem Wettbewerbsmarkt dienten. Der französische religiöse Markt sei durch das Laizitätsprinzip so stark reguliert, dass die Markteintrittsbarriere hoch sei und die Akteure am Markt zu zentralisierenden Aktionen angetrieben seien. Der Slogan

2 Auch Ian Reader (2013) hat in seiner Studie über das Pilgern zu religiösen Stätten in Südostasien die enge Verzahnung von ökonomischen Innovationen zur Sicherung des Einkommens von Tempeln durch Strategien des Marketing herausgearbeitet wie zum Beispiel die Erlebnisanreicherung des Pilgerns über einen Erlebnispark an einem japanischen Flughafen oder die Erschließung säkularer Vertriebssysteme für Pilgerprodukte in Kaufhäusern. 
sei keine Marke, sondern produziere das öffentliche Gut eines toleranten und friedfertigen „gerechten Islams“, für den er seit mehreren Jahre steht. In der Kandidatur sei es um symbolische Kapitalbildung, die muslimische Werte verkörpere, gegangen. Welche Werte das sind, werde von den religiösen Experten festgelegt, die daher zu steuern seien, wenn man den Markt beherrschen wolle. Hanifa Touag beschreibt, wie Salafisten in Frankreich und Belgien ein Ritual als Heilungsangebot aufbereiten, dieses jedoch aufgrund der Ambivalenzen dieser Ökonomisierung unglaubwürdig und letztlich ineffizient wird. Teil III widmet sich der Konsumption: Jochen Hirschle argumentiert, dass mit dem Reichtum einer Gesellschaft zunehmende Konsumaktivitäten auf Kosten der religiösen Aktivitäten gehen. Auch die soziale Interaktion spiele sich mehr und mehr in kommodifizierten Infrastrukturen symbolisch hochwertiger Begegnung in Wellnessressorts oder zu Kulturevents ab und mindere herkömmliches religiös-soziales Engagement. Haytham Siala macht sich Gedanken zur religiösen Markentreue und dem Markenwert. Unterschieden wird die Einstellungs- und die Verhaltensebene für die Kaufentscheidung. Idealerweise geht die erste der zweiten voraus. In ihrer empirischen Studie untersucht sie eine islamische Autoversicherung, die wie jedes Versicherungsprodukt hohe Suchkosten erfordert und riskant ist, um die Stärke der einstellungshaften Markentreue für diese Produktart herauszufinden. Solche empirischen Untersuchungen sind angesichts des stark wachsenden islamischen Finanzmarktsektors in einigen asiatischen Ländern höchst relevant. Schon jetzt werden der christliche Markt der USA auf 7,5 Milliarden US-\$ geschätzt und der muslimische auf 2 Billionen US-\$. Siala kommt zu dem Ergebnis, dass es durchaus eine signifikante Markentreue auf diesem preislich skalierten Sektor gibt, die relativ teuren und riskanten Gütern somit einen religiösen Markenwert zumisst. Diese empirischen Erkenntnisse bergen finanzielle Gewinnchancen in diesem Nischenmarkt. Elizabeth Stickel-Minton belegt empirisch die Korrelation von religiöser Zugehörigkeit und nachhaltigem Konsum und macht einen Vorschlag, wie religiöse Zugehörigkeit jenseits des konfessionellen Modells für korrelierende Marktforschung operationalisiert werden könne.

Teil IV erhebt den Anspruch, eine ökonomische Analyse religiöser Phänomene zu sein, und entfernt sich damit stärker als die übrigen Teile des Bandes von (konsum)soziologischen Überlegungen. Der bereits erwähnte, ausgewiesene Rationalwahlsoziologe Roger Finke schreibt zusammen mit Christopher P. Scheitle wieder einmal, muss man schon sagen - über ökonomische Gründe des religiösen Pluralismus und der Aktivierung von Partizipation. Sie halten daran fest, dass jedwede Restriktion die Partizipation, sprich Nachfrage, mindere. Nachfrage wird nun in Präferenzpluralismus und Konsumpluralismus aufgeteilt. Ersterer geht als Faktor dem Angebotspluralismus vorweg und ist ebenso eigenständig wie der Faktor Bevölkerungsgröße. Denn ab einer bestimmten Bevölkerungszahl teile 
sich der Markt in so viele Nischen, dass sich schon allein dadurch ein Pluralismus einstelle, der allerdings zur Stagnation der Partizipation führe, da aufgrund der vielen Teilmärkte der jeweilige Wettbewerb so gering sei, dass er als Stimulus zu Innovation und Wachstum nicht funktioniere. Für Steve Bruce sind ökonomische Beschreibungen nur für die durch Säkularisierung freigesetzten Märkte des New Age relevant. Religion widersetze sich der Monetarisierung. Eine modelltheoretisch-ökonomische Beschreibung hält er daher nicht für notwendig. Der französische Rechts- und Wirtschaftswissenschaftler Philippe Simonnot, Autor mehrerer Büchern zur „monotheistischen Ökonomie“, Sport, Religion und Kapitalismus (1988, 2005, 2008), stellt das Geschäftsmodell des Jerusalemer Tempels in einer historisch nicht haltbaren Weise vor. Neben seinem ökonomisch reduktionistischen Erklärungsanspruch $(2014,206)$ treffen seine Entwicklungsphasen von einem Gott über ein Volk zu einem Kult für die altisraelitische und frühjüdische Religionsgeschichte nicht zu. Auch Pilatus und Kaiaphas können nicht als Militär und Kirche entgegengesetzt werden (2014, 213), da ihre juridisch-politischen Zuständigkeiten sehr viel komplexer sind, besonders auf der Seite des Hohenpriesters. Dass der Monotheismus das gewinnträchtigste Alleinstellungsmerkmal sei, kann daher nicht überzeugend dargelegt werden.

Mit allen drei Bänden liegen äußerst material- und theoriereiche Sammlungen vor, die unser Wissen über die Anwendbarkeit ökonomischen Wissens für Religion ebenso professionalisieren als auch kritisch diskutieren. Es wird überaus deutlich, dass Religionswissenschaft nicht umhin kommt, Wirtschaft in all ihren Facetten von Ökonomisierung, Kommodifizierung, Konsumgesellschaft und neuen politischen Regulationsformen in ihre Arbeiten einzubeziehen.

In keinem der Bände allerdings wird das Ziel verfolgt, eine systematische disziplinäre Religionsökonomie in Analogie zu Religionssoziologie oder Religionsethnologie $\mathrm{zu}$ entwickeln. Im Gegenteil, in seinem Vorwort $\mathrm{zu}$ „Religions as Brands“ verweist der britische Religionssoziologe David Voas auf Wert und Grenzen der Anwendung von Marketingmodellen auf Religion. Er argumentiert, dass solche Analysen schon allein deshalb notwendig seien, weil Werkzeuge des Marketings von religiösen Organisationen aufgegriffen worden seien, während ökonomische Werkzeuge generalisiert nicht taugten, zum Beispiel zur Erklärung von Islamfeindlichkeit. Voas bestreitet somit genau jene modelltheoretische Reichweite von ökonomischen Konzepten in Gesellschaftstheorien, wie sie andere Soziologen von Bourdieu bis hin zu Luc Boltanski, Eve Chiapello und Axel Paul fruchtbar gemacht haben. Die meisten Beiträge verstehen sich religionssoziologisch oder religionsgeschichtlich, - eine sehr differenzierte Diskussion von Theorien zu Moderne, Konsumption, Gouvernementalität und dergleichen inbegriffen. Durch diesen soziologischen Zugriff besteht leider bei nicht wenigen Beiträgen in allen drei Bänden die Neigung, das Ökonomische nicht-ökonomisch zu erklären 
und erst recht nicht-ökonomische Gegenstände nicht mit ökonomischen Modellen $\mathrm{zu}$ verfolgen. Das ist sehr bedauerlich, vergibt es doch die Chance, neue Methoden und Daten einzubeziehen. Während Religionssoziologien auf der Ebene der Einführungsliteratur mittlerweile sehr kanonisiert sind, mehr noch als Einführungen zum Beispiel in die Religionspsychologie, so gibt es für eine Religionsökonomie bislang noch keine Vorschläge, die sich auch nur vornehmen, diesen Anspruch einzulösen und der Breite der einschlägigen Fragestellungen gerecht $\mathrm{zu}$ werden. So sehr durch die besprochenen Bände Bewegung in das Thema Religion und Wirtschaft gekommen ist, so wenig wird eine Religionsökonomie vorangebracht, solange nicht wirklich wirtschaftssoziologisches und ökonomisches Wissen hinzugezogen wird. Denn schließlich sind Märkte nicht nur soziale Arenen, sondern Arenen, die über Preissetzungen, Produktions- und Vertriebssysteme, vielfältige Marktsicherungsverfahren, also eben über viele spezifisch wirtschaftliche Prozeduren bespielt werden.

\section{Bibliographie}

Brinitzer, Ron. 2003. Religion. Eine institutionenökonomische Analyse. Würzburg: Ergon. Carrette, Jeremy und King, Richard, Hg. 2005. \$elling Spirituality. The silent takeover of religion. London/New York: Routledge.

Eilinghoff, Christian. 2004. Ökonomische Analyse der Religion. Theoretische Konzepte und rechtspolitische Empfehlungen. Frankfurt: Campus.

Hero, Markus. 2010. Die neuen Formen des religiösen Lebens. Eine institutionentheoretische Analyse neuer Religiosität. Würzburg: Ergon.

Illouz, Eva. 2004. Gefühle in Zeiten des Kapitalismus: Adorno-Vorlesungen 2004. Frankfurt: Suhrkamp.

Maddox, Marion. 2013. „Prosper, consume and be saved“. Critical Research on Religion 1: 108-115.

McCleary, Rachel M., Hg. 2011. The Oxford Handbook of the Economics of Religion. Oxford: OUP. Reader, Ian. 2013. Pilgrimage in the Marketplace. London/New York: Routledge.

Schmidtchen, Dieter. 2010. „Ökonomik der Religion“ Themenheft Religionsökonomie. Zeitschrift für Religionswissenschaft 8:11-43.

Simonnot, Philippe. 1988. Homo sportivus: sport, capitalisme et religion. Paris: Gallimard.

Ders. 2005. Les Papes, l'Église et l'argent: histoire économique du christianisme des origines à nos jours. Paris: Bayard.

Ders. 2008. Le marché de Dieu: L'économie des religions monotheists. Paris: Denoël.

Stolz, Jörg. 2008. Salvation Goods and Religious Markets. Bern et al.: Peter Lang.

Vogl, Joseph. 2010. Das Gespenst des Kapitals. Zürich: Diaphanes. 
Miriam Zimmer, Assimilation und religiöse Organisation. Eine organisationssoziologische Studie zu Überlebensstrategien ethnischer Kirchen, Göttinger Junge Forschung 16 (Stuttgart: ibidem, 2013), 186 S., ISBN 978-3-8382-0502-1, € 24,90.

Miriam Zimmer untersucht in ihrer Examensarbeit einen Aspekt zu Minoritäten, der bis dato wenig Beachtung gefunden hat, nämlich die Ausbildung unterschiedlicher Überlebensstrategien ethnisch-religiöser Organisationen, deren Mitglieder sich zunehmend assimilieren. Als empirisches Fallbeispiel ihrer Studie wählte sie die Igreja Evangélica de Confissão Luterana no Brasil, die evangelische Kirche der deutschsprachigen Einwanderer in Brasilien, welche vor allem zu Beginn des 19. Jahrhunderts in das südamerikanische Land kamen. Bildeten in den ersten Jahrzehnten nach der Einwanderung religiös-ethnische Organisationen noch das Zentrum zur Bewahrung ethnischer Traditionen und der jeweiligen Konfession, werden derartige Zusammenschlüsse infolge der Assimilierung ihrer Mitglieder zunehmend überflüssig, wenn sich die Organisation nicht neuen Zielen und Zielgruppen öffnet. Basierend auf dem religionssoziologischen Marktmodell sowie dem Legitimitätskonzept entwickelt die Autorin drei hypothetische Überlebensstrategien, wie ethnische Kirchen auf die zunehmende Assimilierung ihrer Mitglieder reagieren, um das eigene Überleben im religiösen Markt zu gewährleisten. Zur Eruierung der theoretischen Vorannahmen, die jedoch nur für christliche Kirchen aufgrund deren Organisationsstrukturen anwendbar sind (S. 79), dienen 14 leitfadengestützte Experteninterviews aus unterschiedlichen Hierarchieebenen der genannten Kirche. Zum Verständnis der historischen Verknüpfungen fasst Zimmer zunächst die Geschichte der deutschsprachigen Siedler und ihrer Kirche für die letzten knapp 200 Jahre zusammen, um anschließend die Wirkung der innerkirchlichen Reformprogramme der vergangenen Jahrzehnte auf Grundlage der geführten Interviews zu analysieren. Beachtenswert ist ihr Ergebnis, dass Kirchenleitung und -basis unterschiedliche Strategien zur Anpassung ihrer Organisation an die sich verändernden Bedingungen im religiös-deregulierten Markt Brasiliens verfolgen. Zudem verdeutlicht das Ergebnis, dass entgegen der Annahme des Marktmodells, in welchem Laien lediglich als Konsumenten auftreten und Organisationen auf das religiöse Konsumverhalten reagieren, einfache Kirchenmitglieder durch ehrenamtlich wahrgenommene Ämter einen relevanten Einfluss auf das Handeln ihrer Kirche ausüben können (S. 159).

Dirk Schuster: Berlin, Email: dirk_schuster@gmx.de 
Zimmers Arbeit ist eine gelungene Studie, die bezüglich des religiösen Marktmodells auf eine nicht zu unterschätzende Beachtung der Laienpartizipation bei innerkirchlichen Reformprozessen hinweist, wobei die innere Struktur der Kirche bei deren Anpassung an die sich verändernden religiösen und sozialen Gegebenheiten gleichsam von Bedeutung für den Erfolg derartiger Maßnahmen ist. Ebenso zeigt das von ihr gewählte Beispiel, dass die Regulierung bzw. Deregulierung des religiösen Marktes einen direkten Einfluss auf die jeweilige Zielgruppenausrichtung einer kirchlichen Organisation hat, deren Existenz durch die zunehmende Assimilation ihrer Mitglieder bedroht ist.

Thomas Haas, Geistliche als Kreuzfahrer. Der Klerus im Konflikt zwischen Orient und Okzident 1095-1221, Heidelberg transcultural studies 3 (Heidelberg: Winter, 2012), 341 S., ISBN 978-3-8253-6038-2, € 45.

Im Rahmen des Schwerpunktprogramms „Integration und Desintegration der Kulturen im europäischen Mittelalter“ hat Thomas Haas an der Universität Heidelberg die vorliegende Dissertation verfasst. „Wie konnten Geistliche an einem Kreuzzug teilnehmen? Schloss nicht der christliche Glaube Töten und den bewaffneten Kampf aus?“ (S. 9) Wenn hier ein Religionswissenschaftler rezensiert, dann kritisiert er die (fehlende) religionswissenschaftliche Kompetenz in einer historischen Arbeit. Aber sie fehlt empfindlich. Es geht nicht um „den christlichen Glauben“, sondern um die christliche Religion im lateinischen Hochmittelalter. Und da ist die Frage, ob Gewalt das Heil verhindert oder dazu verhilft, unter den zeitgenössischen Theologen sehr umstritten. Weiter: Ein Kreuzzug ist kein Kriegszug; die Teilnahme von Geistlichen ist keine Verpflichtung zum „bewaffneten Kampf“. Die pauschale Behauptung, Papst Urban II. habe die Teilnahme von Geistlichen erlaubt, ist zumindest ungenau. Haas bestimmt zwar die Gruppe der Geistlichen in der Institution, nicht aber ihre soziale Verbindung: Geistliche sind Brüder von Adeligen, von Rittern, von Bauern. Hier wäre erst zu bestimmen: Wer hat alles teilgenommen am Kreuzzug - außer Kriegern und Geistlichen? Ganz besonders der erste Kreuzzug resultiert aus dem Zusammenwirken vieler, sehr verschiedener Gruppen. Unter ihnen gibt es aber grundlegende Differenzen über das Ziel: Die Ritter, darunter die armen zweiten Söhne, die Frankreich bis dahin dauernd in Fehden stürzen, suchen nach einer Burg und einer Frau. Aber sowohl für manche Ritter wie für die anderen Kreuzfahrer steht ein anderes Ziel im Mittel-

Christoph Auffarth: Bremen, Email: auffarth@uni-bremen.de 
punkt: die Pilgerfahrt nach Jerusalem unter militärischem Schutz! Der Kreuzzug ist keine ,bewaffnete Pilgerfahrt', sondern die Verbindung von Pilgerfahrt und Kriegszug, der sich als „Rückeroberung“ legitimiert. Legitimiert durch Geistliche als Historiker. Die Kreuzzugshistoriker sind Geistliche. Alles, was wir an erzählenden Quellen über die Kreuzzüge wissen, ist durch die Perspektive der Geistlichen gefiltert. Haas hat eine umfassende Quellenarbeit mit vielen bisher wenig beachteten Stellen sorgfältig erarbeitet. Bewundernswert ist die Durcharbeitung eines gewaltigen lateinischen Quellencorpus. Keine Frage, in der Sammlung der Quellen liegt der Hauptwert dieser Monographie. Die Quellenarbeit leidet aber darunter, dass Haas sich seine Fragen von den Quellen vorgeben lässt, statt eigene Fragen zu stellen, wie die nach dem Kulturkontakt im globalen Mittelalter, etwa wie sie Michael Borgolte, Nikolas Jaspert, Gerd Althoff, Michael Mitterauer oder auch der Rezensent gestellt haben.

Ausführlicher mit Anmerkungen findet sich diese Rezension wie weitere $\mathrm{zu}$ den Kreuzzügen unter http://buchempfehlungen.blogs.rpi-virtuell.net/

Florian Reiter, Man, Nature and the Infinite. The Scope of Taoist Thunder Magic Rituals 道教雷法, Abhandlungen für die Kunde des Morgenlandes 81

(Wiesbaden: Harrassowitz, 2013), 178 S., ISBN 978-3-447-06904-5, € 48.

Man, Nature and the Infinite ist die zweite Monographie des Autors innerhalb weniger Jahre zur „Donnermagie“ (lei-fa $)^{1}$ des Taoismus, ${ }^{2}$ der einzigen indigenen Hochreligion Chinas. Sie muss im Zusammenhang mit dem 2007 erschienenen Bd. 61 der Abhandlungen für die Kunde des Morgenlandes betrachtet werden, welche sich den Basic Conditions of Taoist Thunder Magic widmet. ${ }^{3}$

Den Kern von Man, Nature and the Infinite bildet die kommentierte Übersetzung der Kapitel 90 bis 92 (von 268) des Corpus of Taoist Rituals (Tao-fa hui-yüan 道法會元, TT 1220) ${ }^{4}$ und eines Essays mit dem Titel „Wan fa t'ung-lun“萬法通論 („Comprehensive Discussion of All Rituals“) des CHANG Shan-yüan 張善淵 (fl. 1280-1294). Zwischen diese zwei Hauptteile mit Übersetzungen ist ein Kapitel gestellt, in dem der Autor zeigt, dass die Donnermagie trotz des Fehlens einer

Michael Hoeckelmann: London, Email: michael.hoeckelmann@kcl.ac.uk

1 Reiter verwendet zur Transkription das Wade-Giles-System, das hier beibehalten wurde.

2 Heute auch häufig „Daoismus“ geschrieben.

3 Wiesbaden: Harrassowitz, 2007.

4 „TT“ steht für (Cheng-t’ung) Tao-tsang, die 1447 erstmals gedruckte, „orthodoxe“ Fassung des taoistischen Kanons. 
einheitlichen Definition kein Sammelsurium, sondern ein einheitliches System bildet. Laut diesem Zwischenkapitel ist die Donnermagie fest in der korrelativen Kosmologie Chinas verankert, die noch heute im Mondkalender, nach dem Feiertage wie das Frühlingsfest datiert werden, den „fünf Wandlungsphasen“ (wuhsing) der TCM u.ä. zum Ausdruck kommt. Diese geht der Entstehung der taoistischen Religion im 2. Jh. n.Chr. voraus, die zuerst wenig mit der Philosophie des Lao-Tzu, Tao Te king, und Chuang-Tzu zu tun hatte. Abgerundet wird das Werk durch Tabellen der auftauchenden Gottheiten, Amulette, ,Zaubersprüche etc.

Der Leser spürt auf jeder Seite, dass Reiter aufgrund seiner langen Erfahrung mit den Texten die Querverbindungen mühelos zu ziehen weiß. Es wäre an mancher Stelle nur hilfreich gewesen, wenn er sie für weniger bewanderte Leser explizit gemacht hätte. Basic Conditions und Scope of Taoist Thunder Magic müssen wohl als ein Werk betrachtet werden, so evoziert das zweite Werk eine Reihe spannender Fragen, für die das erste zumindest mit herangezogen werden sollte: Welche Funktion erfüllte Donnermagie? Wer übte sie wann, wo, wie und wozu aus? Warum sind genau diese Kapitel aus dem Corpus of Taoist Rituals ausgewählt worden? Worin besteht deren insinuierte Beispielhaftigkeit? In Basic Conditions findet der Leser auch einen Abriss der Geschichte, der Methoden und des Anwendungsbereichs der Donnermagie, deren tiefere Wurzeln in der Elitekultur des chinesischen Mittelalters und bei den „Meistern der Esoterika“ (fangshih 方士) des Altertums zu suchen sind.

Man, Nature and the Infinite zeichnet sich durch philologisch exakte Gelehrsamkeit und das Bestreben aus, im Zweifelsfall eine originalgetreue Übersetzung einer leichter zugänglichen, wie sie heute allzu oft im angloamerikanischen Raum $\mathrm{zu}$ finden sind, vorzuziehen. Das Werk leidet ein wenig an einer Formatierung, die am treffendsten als hastig zu bezeichnen ist. So wäre es nicht nötig gewesen, chinesische Schriftzeichen nach jedem Erscheinen eines Namens oder einer Übersetzung in Klammern zu ergänzen (was jedoch dem Fehlen eines umfassenden Index geschuldet sein mag); demgegenüber fehlen die Transkriptionen zwischen Übersetzung und Schriftzeichen in vielen Fällen völlig. Dies mindert gelegentlich ein wenig die Lesefreude, nicht jedoch die hohe inhaltliche Qualität dieser wichtigen Veröffentlichung. 
Christoph Elsas, Hg., Sterben, Tod und Trauer in den Religionen und Kulturen der Welt, Band 1: Gemeinsamkeiten und Besonderheiten in Theorie und Praxis (Hamburg-Schenefeld: EBVerlag, ${ }^{2} 2008$ ), 339 S., ISBN 978-3-936912-2, $€ 19,80$. Band 2: Die Würde des Menschen am Lebensende in Theorie und Praxis (Berlin: EBVerlag, 2011), 413 S., ISBN 978-3-936912-90-6, € 24,80.

Die zwei Bände mit dem Titel „Sterben, Tod und Trauer in den Religionen und Kulturen der Welt“ gehören zu einer Reihe des EBVerlags über das Thema Tod. Der dritte Band dieser Reihe ist ganz dem antiken Ägypten gewidmet und wird hier nicht berücksichtigt.

Der erste Band bietet eine vergleichende historische und religionswissenschaftliche Analyse des Todes in verschiedenen Kulturen. Die ersten zwei Kapitel enthalten methodische Einführungen: Elsas zeigt zunächst, dass der Umgang mit dem Tod heute individualistischer und anonymer geworden ist als in historischen Kulturen wie dem antiken Ägypten. Der Autor schlägt eine dreiteilige Methode, welche Quellen, Vorstellungsbilder und Riten berücksichtigt, vor. In dem zweiten einleitenden Kapitel bemerkt A. Herrmann-Pfandt, dass die religiöse Erklärung des Todes als eine Schwelle zu einer anderen Welt als Trost dient. Der emotionale Aspekt dieses Ansatzes ist wesentlich: Er ermöglicht den Überlebenden, sich die Art der Existenz, die den Verstorbenen erwarten, vorzustellen. Je nach Auffassung der post-mortem-Existenz lassen sich die Kulturen unterteilen: Die einen akzeptieren den Tod, die anderen lehnen ihn ab (S. 58-59). Ab dem dritten Kapitel behandeln die Beiträge den Umgang mit dem Tod in bestimmten Kulturen in mehr oder weniger chronologischer Reihenfolge: antikes China (A. Scottenhammer, D. Schilling); primitiver Buddhismus (B. Analayo); alter Orient (E. von der Osten-Sacken, H. Koch, R. Kessler); antikes Griechenland (H. Froning); Rom (T. Mattern, G. Koch); christlich-europäische Welt (G. Koch, A. Fehrmann, A. Gronemeyer, M. Eidam, S. Glockzin-Bever); in einem abschließenden Kapitel behandelt Elsas den Islam.

Der zweite Band untersucht den Tod im Hinblick auf die Würde des Menschen beim Sterben. Warum der Band erst drei Jahre nach dem ersten erscheint, erklärt Elsas in einer Anmerkung (S. 9). In einem einleitenden Kapitel fasst Elsas die Ergebnisse der Beiträge des Buchs und diejenigen des Unterrichts zusammen, den er im Zeitraum 2007-2010 an der Universität Marburg gegeben hat. M. Greschat schlägt eine Methode vor, die den Tod unter drei chronologischen Gesichtspunkten betrachtet: in der Zeit vor dem Tod, zum Todesmoment und nach dem Tod. In seinem dritten Absatz unterscheidet er je nach Religionstyp (Abstammungsreligi-

Margaret Jaques: Zürich, Email: margaret.jaques@theol.uzh.ch 
on, Wiedergeburtsreligion, Auslöschungsreligion und Auferstehungsreligion) vier verschiedene Reaktionen auf den Tod. Die weiteren Beiträge sind allesamt sehr überblicksartig: eine Theorie der Menschenopfer (A. Herrmann-Pfandt); die Rituale um den Tod bei den Yukpa von Kolumbien (E. Halbmayer), in Afrika (W. Richebächer), in Indien (U. Bulthaup, M. Mittwede), in Tibet (A. HerrmannPfandt, U. Bulthaup) und vom älteren zum jüngeren vorgehend, in den jüdischen, christlichen und muslimischen Religionen (E. Law, T. Mattern, F. Avemarie, A. Standhartinger, C. Werner, T. Anz, U. Brunotte, S. Becker, M. Eidam, A. Marlen Esser und K. Pinggéra).

Das Material des Buchs deckt ein breites Feld ab, sowohl zeitlich als auch methodisch. Eine Zusammenstellung einzelner Beiträge, die alle in der einen oder anderen Weise das Thema Tod behandeln, kann nicht den Anspruch erheben, alle Aspekte abzudecken. Es ist dennoch zu hoffen, dass die Beiträge dieses Buchs weitere wissenschaftliche Forschungen $\mathrm{zu}$ diesem Thema anregen und dass Elsas' Interesse für interdisziplinäre Ansätze und für die Neubewertung herkömmlicher Meinungen in weiteren Erscheinungen dieser Reihe zum Ausdruck kommt.

Klaus E. Müller, Schuld und Sühne. Die Vorgeschichte des Erlösungsglaubens, Wissenschaftliche Paperbacks Ethnologie (Berlin: LIT, 2012), 195 S., ISBN 978-3643117-53-3, € 29,90.

Der Autor, Professor für Völkerkunde, beginnt seine Untersuchung mit einer Erwägung über das Christentum. Dies führt er auf die Überzeugung zurück, dass das Leiden Christi ein einzigartiges und einmaliges Ereignis sei, obwohl auch die altorientalische Tradition Götter kenne, die sterben und zum Leben zurückkehren, wie Dumuzi, Adonis, Osiris und Attis. Der Unterschied zwischen den zwei Glaubenssystemen, dem Christentum und der altorientalischen Tradition, sei nicht gleich dem zwischen Polytheismus und Monotheismus, sondern bestehe besonders in dem Postulat, dass ein einziger Gott dem Volk Israels von den Propheten offenbart wurde. Der Gott von Israel besitze eine absolute Macht, und der Mensch sei Geboten und Verboten unterworfen. Ein Verbot zu verletzen sei eine Sünde, die durch Krankheit, Schmerz, Einsamkeit, Misserfolg und Tod geahndet werde. Auf die Frage „Wie kann dem verurteilten Menschen Vergebung gewährt werden?“ antworte das Christentum mit der Verkörperung Gottes, der alle Sünden auf sich nimmt und sie durch seinen eigenen Tod aufhebt.

Margaret Jaques: Zürich, Email: margaret.jaques@theol.uzh.ch 
In vier Kapiteln, die alle das Stichwort „Martyrium“ enthalten (Martyrium des Bären, Martyrium des Stieres, Martyrium des Menschen, Martyrium von Jesus von Nazareth), analysiert der Autor, wie der Begriff des Opfers, mit der Sünde verbunden, in den Zivilisationen der Welt entstand, um zur Vorstellung von Jesu Opfer zu gelangen. Die Vorgehensweise, in erzählender Form, ist die kritische Erzählung der Lösung der Sünde seit der Vorgeschichte bis zur christlichen Zeit. Sie stützt sich auf ein entwicklungsfähiges Schema, das diesen Gesamtansichten entspricht.

Der Autor unterscheidet zwischen individueller und kollektiver Sünde, kennt aber keine, die, in einem symbolischen System, die Frucht des Zufalles wäre. Der Schuldige, sei er Dämon oder Zauberer, werde zum Ziel eines gelegentlichen oder periodischen Entfernungsrituals: Wiedergeburtsritual nach dem Modell der Durchgangsriten oder Verehrung der Vorfahren. Keinem religiösen Mittel gelinge es aber, die Sünde der Welt aufzuheben. Christus selbst bleibe da erfolglos, und der Mensch müsse seine Hoffnung in die Apokalypse legen, wo das End- und Gesamturteil über die Welt ausgesprochen wird und die Gerechten erlöst werden. Der Autor beschreibt ausführlich die Beispiele, die er zitiert und verweist auf eine reiche, aber eher veraltete Literatur.

Die Analyse, obwohl interessant, ist nicht sehr neu und entspricht einer Betrachtungsweise, die im Christentum, und besonders in der Apokalypse, die Antwort auf das Problem des Übels sieht. Kurz gesagt stellt die Arbeit, dem Titel entsprechend, die Vorgeschichte des Erlösungsglaubens dar.

\section{Rahel Gersch, Frommer Individualismus. Die Lakewood Church und die} Phänomene Megachurch, ,prosperity gospel' und charismatische Pastorenschaft (Berlin: Weißensee, 2013), 324 S., ISBN 978-3-89998-205-3, € 39,80.

Die Lakewood Church im texanischen Houston ist aktuell einer der größten und bedeutendsten Beispiele für das Phänomen einer so genannten „Megachurch“, das ist nach gängiger, primär numerisch orientierter Definition eine protestantische Gemeinde, die pro Wochenende mehr als 2000 Besucher anzieht. Dieses Phänomen wird vor allem mit dem US-amerikanischen Kontext verbunden, obwohl sich in vielen anderen Ländern vergleichbare Erscheinungen finden. Der vorliegenden Monographie kommt das hohe Verdienst $\mathrm{zu}$, sich eingehend mit einem konkreten Beispiel zu beschäftigen und das Phänomen nicht aus einer allgemeinen gesellschaftsanalytischen Perspektive heraus darzustellen. Dabei

Franz Winter: Wien, Email: franz.winter@univie.ac.at 
wird in dieser Mikrostudie aber auch der größere Rahmen nicht außer Acht gelassen.

Das Buch hat zwei Teile, die sich unterschiedlichen Zugängen zur Materie verpflichtet fühlen: Eine historisch orientierte Studie im ersten Teil, wo über die Genese zentraler Begrifflichkeiten und der Church selbst informiert wird, ist mit einer religionssoziologisch orientierten Darstellung im zweiten Teil kombiniert, die einen Einblick in die vielfältigen Lebenswelten der Mitglieder der Lakewood Church geben soll.

Der historische Teilführt die Entstehung der modernen Megachurches und mit ihnen der Lakewood Church sowie der zugrundeliegenden Ideologie der prosperity gospel auf die so genannte revival-Bewegung zurück und zeichnet die Verwurzelung im Kontext der puritanischen Theologie detailliert nach. Damit sprengt die Arbeit die gängige Darstellung und spannt einen viel weiteren Bogen, der sich um eine Einbettung in die Geschichte des US-amerikanischen Christentums bemüht. Allerdings scheint der religionshistorische Blick zuweilen zu sehr auf die Entwicklung innerhalb der unterschiedlichen christlichen Filiationen in den USA fokussiert zu sein. So fehlt beispielsweise eine Thematisierung der Bedeutung der New Thought-Bewegung gerade für Grundideen der prosperity gospel, womit in einem größeren Ausmaß die Interdependenz unterschiedlicher weltanschaulicher Bezugsfelder deutlicher gemacht würde.

Der zweite Teilfußt auf einer Rezipientenstudie, die zwischen 2009 und 2011 in der Lakewood Church vor Ort ausgeführt wurde. Im Fokus stand das Anliegen, ein besseres Verständnis der Perspektive derer zu erlangen, die am Leben der Church partizipieren und sich von ihr angesprochen fühlen. Besonderes Augenmerk wird hier auf die Bedeutung und die Stellung des aktuellen Leiters der Lakewood Church, Joel Osteen, gelenkt, dessen „charismatische Pastorenschaft“ als eine der bedeutenden Faktoren für den aktuellen Erfolg herausgearbeitet wird. Mit der Einzelanalyse gelingt es dem Autor gut, klassische Klischees zu durchbrechen, die man gemeinhin mit dem amerikanischen protestantischen Milieu der Megachurches und den Rezipienten einer prosperity gospel verbindet. Vielmehr fällt die Vielfalt der möglichen Motivationspunkte bei den Befragten auf, die zudem einen hohen Grad an Individualismus erkennen lassen. Damit kann die hierzulande oftmals gängige Sortierung dieses spezifischen Phänomens zumindest in Frage gestellt werden.

Mit der veranschlagten Zweiteilung gelingt eine umfassende Gesamtdarstellung des Phänomens Lakewood Church und der ihr zugrundeliegenden Rahmenbedingen, die beispielgebend sein könnte. Die Kombination aus detaillierter historischer Aufarbeitung, die für sich schon gewinnbringend zu lesen ist, und einer religionssoziologischen Analyse der aktuellen Situation gibt einen umfassenden Einblick. Formal heftig zu kritisieren ist das Fehlen eines Index, der ein 
unerlässliches Instrument zur sinnvollen Erschließung einer akademischen Arbeit ist und bleiben sollte - wenn die Erstellung eines solchen bekanntermaßen auch noch so mühselig ist.

\section{Christiane Brosius, Axel Michaels und Paula Schrode, Hg., Ritual und} Ritualdynamik (Göttingen: Vandenhoeck \& Ruprecht, 2013), 240 S., ISBN 978-3-8252-3854-4, € 24,99.

Der Sammelband ist das Kind des zehnjährigen Wirkens der Mitglieder des Sonderforschungsbereichs 619 „Ritualdynamik“. Das Uni-Taschenbuch bietet komprimierte Fachinformationen zu prominenten Begriffen der Ritualforschung und strahlt in verschiedenste Disziplinen aus. Neben der Vorstellung der Beiträge umreißt die Einführung zunächst die Entwicklung und Kontextualisierung der akademischen Verwendung des Ritualbegriffs und zeigt dessen Variabilität und Komplexität auf. Laut der eingangs formulierten Intention soll die Multiperspektivität helfen, Rituale und ihre kulturellen Prägungen sowie ihre Wandlungen auf verschiedenen Ebenen zu erkennen.

Das Inhaltsverzeichnis im Stil eines Sachwörterbuchs umfasst neben einem allgemeinen Überblick 27 Stichworte. Die zugeordneten Artikel gliedern sich in Begriffs- bzw. Theoriegeschichte, Diskussion und Forschungsbeispiele, wobei v.a. die Literaturverzeichnisse mit Empfehlungen zur einführenden Lektüre hilfreich sind. Verknüpft sind die Beiträge durch Querverweise. So kann der Leser erkennen, welche Schlüsselbegriffe inhaltlich und zeitlich im Rahmen der Ritualforschung verwandt sind. Davon können hier nur einige exemplarisch vorgestellt werden, welche sich leicht miteinander ins Gespräch bringen lassen:

W. S. Sax erklärt, dass entgegen der unter Sozialtheoretikern häufigen Verwendung des Begriffs Agency als Widerstand von Individuen gegen eine herrschende Gesellschaft darunter vielmehr eine Potenz zur Veränderung durch Einzelne, ein Kollektiv, Institutionen, Praktiken oder nicht-menschliche Wesen zu verstehen sei. Damit rege der Begriff Fragen zur Autorschaft und Funktionsweise sowie zur Transformationskraft von Ritualen an, welche die Handlungspotenz manifest werden ließen. Davon zu unterscheiden ist die Bezeichnung Ritualmacher, welche laut J. Gengnagel und G. Schwedler die ausführenden Träger einer rituellen Handlung meint. Ihrer Rolle nähern sich die Autoren durch Titel und Ämter, die „Zeremonienmeistern“ zukommen. Zur Wirksamkeit von Ritualen kritisiert P. Töbelmann die häufig vorgenommene Wahr-Falsch-Unterscheidung, was

Jenny Vorpahl: Potsdam, Email: jenny.vorpahl@uni-potsdam.de 
die Effektivität von Ritualen aus Sicht der Wissenschaftler und der Teilnehmer betrifft. Gleichzeitig gesteht er ein, dass eine objektive Feststellung von Wirksamkeit nicht erreichbar sei, sondern nur ein möglichst differenzierter Zugang.

Nach einem Abtasten der Bedeutungen von Ästhetik wenden sich T. Graf und I. Prohl der Fruchtbarmachung dieser Kategorie für die religionswissenschaftliche Ritualforschung zu, indem sie Theorieansätze anreißen, die von einem rein idealisierenden Verständnis absehen und das kulturell geprägte sinnliche Wahrnehmen und Erkennen hervorheben. Betont wird das Feld der „Material Studies“, wobei interessiert, wie in Ritualen verkörperte Religion die Sinneswahrnehmung reizt und lenkt. Eng damit verbunden sind als Transportmittel von Deutungen religiöse Medien, welche T. Meier und A. Zotter im Artikel zu Ritualgegenstände und Materialität aufgreifen. C. Brosius und S. Heidbrink definieren Medialität als das Bedingen von Handlungen durch Medien im Hinblick auf die Produktion, Zirkulation und Rezeption von Ritualen. Gemeint sind Massenmedien, welche ihre Bedeutung erst durch ihre Verwendung gewännen, deren Wirkung sich u. a. in der Informationsvermittlung und der Verbindung zwischen Ritualakteuren entfaltet. Daraus ergeben sich Fragen nach Erhalt, Transfer, Wiederholbarkeit und Öffentlichkeit von Ritualen sowie nach der Ritualautorität und realer Teilnahme.

An die ästhetischen und medialen Aspekte schließt das von K. Polit erläuterte Konzept von Verkörperung an. Entgegen der Körper-Geist-Dichotomie geht es ihr zufolge nicht nur um einen erlernbaren Habitus oder um den Körper als Ausdrucksmittel und Ritualobjekt, sondern um die Sinnhaftigkeit und identitätsstiftende Handlungsmacht von Körpern sowie die kulturell beeinflusste Verkörperung von Erfahrung, Emotionen, Wissen und Interpretationen im Kontext ritueller Handlungen. C. Ambos und J. Weinhold nehmen die Entstehung und Funktion von Rahmen und Rahmungsprozessen für das Wahrnehmen von Ritualen in den Blick. Erlernte, im Rahmen gesetzte kommunikative Signale dienen der Anleitung von Handlungen, Emotionen und Interpretationen. Besonders erhellend ist hier die Auseinandersetzung mit der Koexistenz sowie der Instrumentalisierung verschiedener Rahmen. Ergänzend empfiehlt sich z.B. die Lektüre des Beitrags zu Ritualdesign von G. Ahn, N. Miczek und C. Zotter, da er sich mit der bewussten Gestaltung von Ritualen und deren Legitimierung durch tradierte Ritualelemente einem populären Feld sowohl der Ritualpraxis als auch deren Erforschung zuwendet. Als ähnlich aktuell und noch wenig bearbeitet stellt U. Hüsken die Analyse von Ritualfehlern und ihren Konsequenzen als Subkategorie des Begriffs Ritualkritik vor, welchem sie an späterer Stelle in Zusammenarbeit mit R. L. Grimes auf den Grund geht. In beiden Fällen sind die dem Scheitern bzw. der Bewertung zugrundeliegenden Normen, Motivationen, Handlungskompetenzen, Perspektiven und Konsequenzen zentrale Analysefaktoren, sodass sich neue Möglichkeiten des Vergleichs eröffnen. 
Auffällig ist, dass fast alle Beiträge mit dem Begriff Ritualdynamik in Beziehung gesetzt werden können, mit dessen Genese und Entwicklung sich D. Harth und A. Michaels separat auseinandersetzen, sodass sich der Titel des Bandes erklärt. Die einzelnen Stichworte zeigen, auf wie vielen Ebenen sich Modifikationen durch und in Ritualen ergeben und aus welchen Perspektiven diese beobachtet werden können. Eine andere Möglichkeit des Aufbaus wäre eine sachliche Anordnung der Begriffe gewesen, z.B. entlang der Entwicklung der Ritualforschung oder zu semantischen Feldern gruppierte Stichworte. Gerade die Ausführungen zu Ritus/Ritual von J. Quack sind ein geeigneter Einstieg, wenn man das Handbuch nicht nur zum Nachschlagen verwenden, sondern zusammenhängend lesen möchte. Die Aktualität sowie der inflationäre Gebrauch des Begriffs „Ritual“ haben die Zusammenstellung eines solchen Bandes erforderlich gemacht. Gerade weil die aufgeführten Stichworte immer wieder in Populär- und Fachliteratur auftauchen, synonym verwendet oder unterschiedlich verstanden werden oder gar unreflektiert bleiben, füllt dieses Werk eine auffällige Lücke im Bücherregal eines Ritualforschers und interessierten Lesers.

Tarjei Rønnow, Saving Nature. Religion as Environmentalism, Environmentalism as Religion, Studies in Religion and the Environment 4 (Berlin u. a.: LIT, 2011), 288 S., ISBN 978-3-643-11052-7, € 29,90.

„Saving Nature“ war als Dissertation gedacht und ist posthum erschienen an der Fakultät für Geisteswissenschaften an der Norwegischen Universität für Wissenschaft und Technologie in Trondheim. Der Autor war Wissenschaftler im Bereich Religious Studies. Seine zentrale These ist: Während Religion (nämlich die christliche) im Bereich des Umweltschutzes wirken kann, kann profaner Umweltschutz ebenso als Religion wirken.

Seine Doktorarbeit gliedert Rønnow in drei Hauptteile: The Greening of the Church, The Environmental Movement und The New Pietism. 13 Seiten Quellenangaben runden sein Werk ab.

Der erste Teil behandelt das Aufgreifen ökologischer Themen durch die Kirche (,grüne/r“ Kindergottesdienst/ Konfirmandenunterricht/ Erwachsenenbildung; „grüne“ Hymnen/ Gebete/ Psalmen etc., d.h.: Auffinden von Deckungsgleichheiten von biblischer Tradition und den Forderungen des Umweltschutzes in der Theorie) sowie das Umsetzen in die Praxis, z.B. durch nachhaltiges Gemeinde-Management, Zusammenarbeit mit profanen Umwelt-Organisationen

Sylvia Paetzold-Siewert Rheinbach, Email: Sylvia.Paetzold-Siewert@t-online.de 
bis hin $\mathrm{zu}$ weitgefassten Organisationen wie dem World Council of Churches (WCC) mit dem Programm „Justice, Peace and the Integrity of Creation“ (JPIC) oder der Alliance of Religion and Conservation (ARC) mit vielen Mitgliedern auch nichtchristlicher Glaubensrichtungen. Den Schwerpunkt seiner Untersuchungen legt Rønnow auf die Entwicklung in Norwegen, daher gipfelt der erste Teil in der Beschreibung der Korsvei-Bewegung, die aus einer Sammlung unterschiedlicher Bewegungen 1984/1985 entstand und im Zusammenhang zu sehen ist mit dem norwegischen Zweig der charismatischen Jesus-Bewegung.

Im zweiten Teil stellt Rønnow dar, wie schwer es ist, eine treffende Definition für „Environmentalism“ zu finden; wenn man dieses als Diskurs oder kulturellen Prozess betrachte, so könne es in Erscheinung treten als (gesellschaftliche) Bewegung, als (politische) Ideologie sowie als Wiederbelebung religiöser Traditionen (S. 124). Im weiteren Verlauf gibt der Autor einen kurzen Überblick über die Entwicklung der Umweltschutz-Bewegung in Norwegen sowie eine soziologische Einordnung in die Kette Religion-Säkularisation-Resakralisierung. Rønnow findet in der Entwicklung der norwegischen Umweltbewegung, ähnlich wie in anderen westlichen Ländern, religiöse Elemente - z.B. bei der Verwendung und Deutung christlicher Symbole und Termini (S. 244ff. u.a.).

Hier sind wir beim dritten Teil angekommen, in dem die (ökologische) Erweckung, die Natur als Heiliges und der Pietismus thematisiert werden. Rønnow zeigt auf, wie die Natur als Urkonzept des Heiligen (S. 219) mit den klassischen Konzepten „Heiliger Ort, Heilige Geschichte, Heilige Zeit“ und Transzendenz zusammengebracht werden kann. Weil Norwegens Religionsgeschichte in den letzten 200 Jahren von pietistischen Bewegungen geprägt wurde (S. 241), flossen deren topoi in die Umweltbewegung ein: „It would seem that simply by switching the words ,sin' with ,consumption“ and ,God' with ,nature‘, we arrive at an anthropology that lies very close to the current environmental gospel“"(S. 247).

Rønnow hat in seiner Arbeit aufgezeigt, wie in Norwegen Religion (nämlich die christliche) und Umweltschutzbewegung ineinanderfließen, oder, wie er es nennt, „interconnected“ (S. 259) sind. Während die Kirche ökologische Themen aufgreife, auch um sich selbst in der modernen Welt zu legitimieren, greife die Umweltbewegung christliche Symbole und Termini auf und eigne sich so die moralische Durchschlagskraft einer Religionsgemeinschaft an.

Seine Aufgabe, die Beziehung zwischen Umweltbewegung und Religion (nämlich der christlichen) in Norwegen zu untersuchen, hat Rønnow ausführlich und interessant gelöst. Die Zitate lockern den Textfluss auf und sind gut ausgewählt, um Sachverhalte auf den Punkt zu bringen. Einziger Kritikpunkt: Ein kleines Glossar für die Abkürzungen wäre hilfreich gewesen. 
Martina Dürkop, Das Archiv für Religionswissenschaft in den Jahren 1919 bis 1939. Dargestellt auf der Grundlage des Briefwechsels zwischen Otto Weinreich und Martin P:n Nilsson, Religionswissenschaft 20 (Berlin/Münster: LIT, 2013), 506 S., ISBN 978-3-643-11129-6, € 49,90.

Im Zuge der beginnenden Emanzipation der Religionswissenschaft gegenüber der Theologie als einer eigenständigen universitären Fachrichtung gründete Thomas Achelis 1898 das Archiv für Religionswissenschaft (ARW). Der wenige Jahre später beginnende Erste Weltkrieg sollte für die gesamte deutsche Wissenschaftslandschaft eine einschneidende Zäsur darstellen, von der ebenso das ARW betroffen war. Nicht nur der Kontaktabbruch zu Kollegen aus den Ländern, die gegen das Kaiserreich im Krieg standen, sorgte für eine zunehmende Isolierung deutscher Gelehrter. Ebenso machten die mit fortschreitender Kriegsdauer einsetzende politische Orientierung neutraler Staaten wie Schweden in Richtung der westlichen Alliierten einen fachlichen Austausch über die deutschen Grenzen hinweg immer schwieriger. Dass sich diese Situation nach der Kriegsniederlage nicht grundlegend änderte, verdeutlicht Martina Dürkop in ihrer Studie, einer leicht überarbeiteten Version ihrer 2009 an der Universität Erfurt eingereichten Dissertation. Wie Titel und Untertitel darlegen, wird die Geschichte des ARW auf Grundlage der Korrespondenzen der beiden Herausgeber nachgezeichnet, wobei die Entwicklung des ARW in den wissenschaftsgeschichtlichen sowie zeithistorischen Kontext eingebettet wird. Den Briefwechsel der beiden Herausgeber, soweit noch überliefert, gibt Dürkop im zweiten Teil des Buches auf rund 200 Seiten in edierter Form wieder. Da mit Martin Persson Nilsson einer der beiden Herausgeber des ARW im untersuchten Zeitraum ein Schwede mit Lehrstuhl in Lund war, nimmt Dürkop vor allem die Wissenschaftsbeziehungen zwischen Deutschland und Schweden in den Fokus ihrer Darstellung. Zur klareren Orientierung skizziert sie hierzu in den ersten beiden Kapiteln die schwedische Wissenschaftsentwicklung im internationalen Kontext für die Zeit vor 1914. Die zwei anschließenden Kapitel widmen sich den Rahmenbedingungen und Einschränkungen, mit denen sich deutsche und schwedische Forscher ab 1914 konfrontiert sahen, und den Folgen, die der Wissenschaftsboykott gegen Deutschland ab 1919 mit sich brachte.

Noch während des Ersten Weltkrieges entschied man sich seitens des ARW, ausländische Mitarbeiter in die Redaktion aufzunehmen und richtete eine entsprechende Anfrage an Nilsson, der bereits zuvor als Autor im ARW in Erscheinung getreten war. Infolge des Absatzeinbruches während des Krieges und der

Dirk Schuster: Berlin, Email: dirk_schuster@gmx.de 
allgemein zunehmenden finanziellen Verluste der Verlage bei dem Vertrieb wissenschaftlicher Zeitschriften als Resultat der immer weiter ausufernden Inflation in Deutschland war die weitere Finanzierung des ARW nach Kriegsende in ständiger Gefahr. Neben verschiedenen Zuschüssen aus dem In- und Ausland unterstützte die Stockholmer Religionswissenschaftliche Gesellschaft das ARW in den 1920er und 1930er Jahren fortlaufend, was auf das noch immer große Interesse der Zeitschrift im Ausland zurückzuführen ist, auch wenn es wegen des Wissenschaftsboykotts gegen Deutschland immer schwieriger wurde, die Absatzzahlen im Ausland auf konstantem Niveau zu halten. Aufgrund der schwachen finanziellen Grundlage sowie zur zahlenmäßigen Sicherung der ausländischen Bezieher vereinigte man 1923 das ARW mit den Beiträgen zur Religionswissenschaft der Religionswissenschaftlichen Gesellschaft in Stockholm. Die dadurch eintretende Stabilisierung bezüglich Finanzierung und Abonnements sorgten für eine ruhigere Fortentwicklung des ARW in der zweiten Hälfte der 1920er Jahre, obwohl die Zeitschrift auf weitere Zuschüsse angewiesen blieb (S. 129). Auch wenn in dieser Zeit zunehmend Beiträge von schwedischen Wissenschaftlern beim ARW eingereicht wurden, blieb dennoch eine sich ausweitende wissenschaftliche Zusammenarbeit zwischen deutschen und schwedischen Religionswissenschaftlern aus.

Nach 1933 vollzog der zweite Herausgeber, Otto Weinreich, genauso wie viele seiner Berufskollegen, eine Anpassung an die neuen politischen und gesellschaftlichen Gegebenheiten. So lehnte Weinreich noch 1926 eine Arbeit zur altgermanischen Religionsforschung ab, forderte diese aber im September 1933 plötzlich für das ARW ein (S. 204). Neben den zunehmenden Kontaktverlusten zu ausländischen Wissenschaftlern als direkte Folge der nationalsozialistischen Politik mischten sich überdies staatliche Stellen in immer stärkerem Maße in die Wissenschaft ein, unter anderem in das wissenschaftliche Zeitschriftenangebot (S. 215). Leider geht die Autorin auf diesen wichtigen Punkt nicht näher ein, weshalb dem Leser vorenthalten bleibt, ob dies bereits Mitte der 1930er Jahre Auswirkungen auf das ARW haben sollte. Ebenso im Unklaren bleibt, wieso die Absatzzahlen der Zeitschrift zwischen 1929 und 1936 von 404 (1929) auf 272 Bezieher (1936) - ein Rückgang um mehr als 35\%! - einbrachen, wie Dürkop lediglich in einer Statistik aufzeigt (S. 235).

Mit dem Herausdrängen von Weinreich aus der Herausgeberschaft infolge des zunehmenden Einflusses des SS-Ahnenerbes beendete auch Nilsson sein Engagement für das ARW, ebenso der zwischenzeitlich durch den Verlag installierte dritte Herausgeber Friedrich Pfister. An dieser Stelle fehlt eine Überblicksdarstellung über die weitere Entwicklung bis zur Einstellung der Zeitschrift 1942, die von einer starken Politisierung der Beiträge im Sinne des SS-Ahnenerbes geprägt war. Denn dies stellte einen der Hauptgründe dar, warum diese traditi- 
onsreiche Zeitschrift nach dem Kriegsende 1945 nicht wiederbelebt wurde, was der Leser auf den entsprechenden Seiten jedoch nicht erfährt (S. 254f.).

Es bleibt ein etwas zwiespältiger Eindruck bei der Lektüre dieses Buches: Einerseits bietet die Untersuchung der Geschichte des ARW auf Grundlage der Herausgeberkorrespondenzen Einblicke in die Hintergründe mancher Entscheidungen und deren Folgen. Mit der Darstellung der wissenschaftlichen Beziehungen zwischen Deutschland und Schweden auch über die reinen Fachgrenzen der Religionswissenschaft hinaus, verdeutlicht die Autorin, wie eng die internationale Zusammenarbeit im Untersuchungszeitraum war. Gleichsam wird deutlich, welch hohe Reputation das ARW in der internationalen Fachwelt genoss. Andererseits verliert sich die Autorin nicht selten in der bloßen Wiedergabe von Zahlen über Auflage, Kosten etc., ohne diese jedoch in irgendeinen Zusammenhang zu stellen und daraus allgemeinere Aussagen abzuleiten. Geht die Autorin immer wieder auf die Situation der schwedischen Religionswissenschaft in deren Ringen um internationale Anerkennung ein, wofür sie umfangreich auf schwedische Forschungsliteratur zurückgreift, fehlt andererseits die Bezugnahme auf die deutsche Fachgeschichte fast gänzlich, was vor dem Hintergrund des Themas verwunderlich ist. Es ist ohne Zweifel wichtig und unabdingbar, die Geschichte des ARW mit Hilfe der brieflichen Hinterlassenschaften der Herausgeber zu rekonstruieren. Diese vollzog sich aber nicht in einem luftleeren Raum, sondern in direkter Beziehung zu der sich etablierenden und ausdifferenzierenden Religionswissenschaft als akademisches Fach, deren wichtiges Mitteilungsorgan das ARW darstellte. Durch den ausbleibenden Rekurs auf die religionswissenschaftliche Fachgeschichte in Deutschland für den untersuchten Zeitraum bleibt die Arbeit leider unvollständig.

Peter J. Bräunlein, Zur Aktualität von Victor W. Turner. Einleitung in sein Werk. Aktuelle und klassische Sozial- und Kulturwissenschaftler/innen (Wiesbaden: Springer, 2012), 187 S., ISBN 978-3531169071, € 22,99.

Victor Turner gehört zu den Klassikern der Ritualtheorie. Peter Bräunlein stellt eindrücklich in der „Einleitung in sein Werk“ die Genese und Wirkung seiner vielfältigen Arbeiten vor, durch die aber immer ein roter Faden geht. Die Konzepte communitas, Liminalität, soziales Drama und Erleben seien als Versuch Turners zu sehen, „dem Rätsel des Humanum in Ritual und Spiel, in Kunst und Religion, im Drama des Lebens selbst nachzuspüren“ (S. 150). Konsequent im gesamten

Katharina Wilkens: München, Email: katharina.wilkens@lrz.uni-muenchen.de 
Band ist die Präsenz von Edith Turner sichtbar gemacht. Das vorletzte Kapitel geht dementsprechend gezielt auf ihre eigenständige Arbeit nach Victor Turners Tod ein. Im Buch werden die Turners als Menschen in zurückhaltend humorvoller, sympathischer und lebendiger Weise heraufbeschworen, sodass ein Gleichgewicht zwischen Theorien und persönlichen Interessen entsteht - die oft genug den strengen Regeln der Akademie widersprachen.

Die zwölf Kapitel der Einführung sind dem Werk Turners folgend chronologisch gegliedert und damit zugleich thematisch im Kontext zeitgenössischer akademischer Entwicklungen verortet, mit denen sich Turner jeweils auseinandersetzte. Im Kontext des Frühwerks entfaltet Bräunlein umfassend den Hintergrund des Strukturfunktionalismus der 50er und 60er Jahre und Turners eigener interpretativen und performativen Wende. Bei der Diskussion des Spätwerks wird die ethnologische Krise der 80er Jahre zwischen der Suche nach Authentizität, experimenteller Performance und postmoderner Selbstverwirklichung detailreich und hintergründig erläutert. Immer wird die zunächst marxistische, später konservativ-katholische Innenperspektive der Turners in ein Verhältnis zu den theoretischen Konzepten gesetzt. Besonders hervorzuheben ist Bräunleins Geschick, in Exkursen immer wieder das aktuelle politische und teils auch private Geschehen $\mathrm{zu}$ entfalten und unmittelbar auf die Schriften und Projekte von Turner zu beziehen.

Insgesamt hält sich Bräunlein mit einer Bewertung des Turner'schen Werks weitgehend zurück. Erst im letzten Kapitel werden systematisch die vielfältigen und durchaus gewichtigen Kritikpunkte zusammengeführt, die in der Rezeption seines Werks hervorgebracht wurden, und mit Turners eigenem, interdisziplinären Werkverständnis kontrastiert. Gerade der offensichtlich theologisch-philosophische Einschlag von Turner und sein Bemühen um eine kulturelle Wirksamkeit seiner Thesen jenseits der akademischen Ethnologie (beispielhaft sei hier nur das Konzept der communitas genannt) haben jedoch seine breite Nachwirkung befördert, sodass eine Auseinandersetzung mit diesem „kreativen“ und „rastlosen“ Autor (S. 9f.) - auch und gerade für ReligionswissenschaftlerInnen - unabdingbar bleibt, so das Fazit von Bräunlein.

Zwar spricht das Buch vor allem den ethnologischen Entstehungshintergrund von Turners Werk an. Trotzdem werden viele Themen und Fragen aufgeworfen, die auch für ReligionswissenschaftlerInnen relevant sind (weshalb Bräunlein Turner bereits in Axel Michaels’ Sammelband „Klassiker der Religionswissenschaft“ vorgestellt hatte). Dazu gehört insbesondere auch eine kritisch-reflexive Analyse der Entwicklung von Religionstheorien. In Bräunleins Darstellung wird beispielhaft der Entstehungsprozess von Studien wie Chihamba, Image and Pilgrimage sowie dem Spätwerk, das in der Neurotheologie mündet, eindrücklich in Beziehung zur religiösen Biographie der Turners gesetzt. So kann die Konzeption 
einer erfahrungsbasierten, mystischen Religion, die sich in Ritualen aktualisiert, im zeitgenössischen Umfeld - zum Teil gegen den Zeitgeist - verortet werden.

In diesem Sinne ist dieses Buch von Peter Bräunlein zum nach wie vor aktuellen, religionswissenschaftlichen Klassiker Victor Turner unbedingt empfehlenswert.

\section{Steven J. Sutcliffe und Ingvild Sælid Gilhus, Hg., New Age}

Spirituality - Rethinking Religion (Durham: Acumen Publishing Ltd., 2013), 320 S., ISBN 978-1844657148, f19.99/€ 26,38

Die im Herbst 2013 erschienene und insgesamt fünfzehn Beiträge umfassende Anthologie New Age Spirituality - Rethinking Religion ist seit langer Zeit die erste umfassende Publikation, die sich dem Thema „New Age“ beziehungsweise den New Spiritualities widmet. Die Autoren, renommierte Religionswissenschaftler, Soziologen und Anthropologen, stellen mögliche zukünftige Forschungsperspektiven auf den Gegenstand vor: Vor allem soll sich nach einer Welle von Fallstudien hier dem theoretischen Potenzial der oft als marginal angesehenen New Spiritualities als einer typischen Form von Religion zugewendet werden. Die durchaus begründete Annahme, eine Inklusion der New Spiritualities könne zur Entwicklung von generellen Theorien über Religion beitragen, stellt hernach einen wichtigen roten Faden des Sammelbandes dar.

In der Einleitung konzeptualisieren Sutcliffe und Gilhus vor dem Hintergrund des Forschungsstandes, vor allem der englischsprachigen Literatur und vielen der bisherigen Ansätze entgegen, die New Spiritualities als „mixed and fluid“. Die zur Verfügung stehenden Daten seien eng mit verschiedenen weiterreichenden Prozessen in westlichen Gesellschaften - Globalisierung, Pluralisierung, Individualisierung, Säkularisierung, Re-Sakralisierung sowie Kapitalismus und Mediatisierung - verwoben und würden dementsprechend auch in nicht-religiösen Sektoren produziert (S. 7f). Die New Spiritualities sind also als inhärent dynamisch und die Grenzen bisheriger epistemologischer Taxonomien überschreitend zu verstehen, während die „orthodoxen“ soziologischen Foci auf institutionalisierte Religion und formative religiöse Autorität im Zentrum der Kritik stehen: Nicht die scharf abgegrenzten Formen von Religion sind den Autoren zufolge „normal“, sondern vielmehr gelebte Religion (lived religion) alltäglich und weitreichend präsent. Die New Spiritualities und die folk religion würden vielmehr

Christiane Königstedt: Leipzig, Email: c.koenigstedt@uni-leipzig.de 
Beispiele für den normalen Aggregatzustand von Religion darstellen als deren sehr viel öfter besprochenen - stark institutionalisierte Formen (S. 12f).

Die Aufsätze der Anthologie sind in drei Abschnitte gegliedert. Im ersten wird eine Evaluierung der Funktionalität genereller Theorien und Konzepte von Religion in Bezug auf die New Spiritualities unternommen: Diskutiert werden die New Spiritualities unter anderem als Durkheims „elementare Formen von Religion“ sowie im Rahmen von Jonathan Z. Smith' spatial model of religion und als Bensons Salers „prototypische Religion“. Hierbei wird deutlich, dass eine Integration des Gegenstandes die Modelle bereichern und Empirie-gerechtere Analysen ermöglichen kann. Anschließend wird die These der Möglichkeit einer sich selbst genügenden säkularen Gesellschaft sowie deren Gegenteil, die über deren Insuffizienz geprüft. Im letzten Aufsatz des ersten Teils werden die Organisationsformen des „New Age“ zwischen soziologischen Konzepten und anti-autoritären und anti-hierarchischen Präferenzen aus der Selbstbeschreibung des Milieus neu und kritisch besprochen.

Die New Spiritualities werden im zweiten Abschnitt im weiteren Kontext von Religion und Kultur und im Vergleich verschiedener Länder behandelt, angefangen mit einem flexiblen Modell von „Spiritualität“ wird das „New Age“ mittig auf den Achsen religiös/nicht-religiös und lokal/global sowie im Zusammenhang mit volksreligiösen (folk religiosity-) Revivals verortet. Es folgen Analysen verschiedener regionaler kultureller und religiöser Kontexte (Brasilien, Norwegen, Polen), mit denen die New Spiritualities erkennbar verwoben sind. Abgeschlossen wird dieser Abschnitt mit einer Herausstellung der Konzepte und Werte im „New Age“, die, so die Autorin, zentral für dessen marktförmige Entwicklung in der Konsumgesellschaft seien.

Im dritten Abschnitt werden die gegenwärtige gesellschaftliche Präsenz und Funktionsweisen der New Spiritualities aus verschiedenen Perspektiven behandelt, von „New Age“-Organisationen in den Niederlanden bis zu deren explizit formativen Wirkungen auf das menschliche Verhalten am Beispiel des spirituellen Milieus Taiwans. Mehr im Kontrast hierzu steht ein Beitrag, der sich mit dem Konzept der „kognitiv optimalen Religion“ wieder der individuellen Glaubenserfahrung zuwendet. Ergänzend wird folgend die Entwicklung des Feldes in den Niederlanden seit den 1960ern nachgezeichnet und eine Typologie religiöser und säkularer Spiritualität vorgeschlagen, die m.E. eine mögliche Brücke zwischen der „fluiden“ Konzeptualisierung der New Spiritualities und anderen Ansätze schlagen könnte. Der letzte und vielleicht innovativste Beitrag des Bandes fragt nach der Bedeutung der innerhalb von „New Age“-Praktiken konstruierten agents (z.B. Engeln) für die von den Praktizierenden wahrgenommene Lebenswelt.

Zusammen betrachtet stellen die Aufsätze nicht nur einen hochinteressanten Fundus an Ergebnissen und Perspektiven auf die New Spiritualities dar, sondern 
setzen diese auch erstmals so ausgiebig in Relation zu anderen Formen von Religion, sowie größeren kulturellen Strömungen und identifizieren vermehrt Orte ihrer gesellschaftlichen Relevanz. Der Gegenstand selbst gewinnt damit zumindest analytisch stark an Kontur, und darüber hinaus gelingt es den Autoren unter Verwendung modifizierter klassischer und selbst entwickelter Ansätze zu zeigen, wie die Vielgestaltigkeit der New Spiritualities bestehende Theorien über Religion infrage stellt und damit Anregungen zu deren Ergänzung liefert. Ihr Fazit ,[the New Age] is... good to think!" begründet sich weiter vor allem in ihm als Alternative zum Weltreligionen-Paradigma, nicht zuletzt aber auch durch den an vielen Stellen explorativen Charakter des Disziplinen-übergreifenden Unterfangens. Gegenüber älteren Theorien, die das „New Age“ als Form von Religion betonten (wie Thomas Luckmanns „Unsichtbare Religion“), zeichnen sich die vorliegenden Aufsätze vor allem durch ihre starke empirische Fundierung aus. Anzumerken ist, dass, während sich einige wichtige Forschungsfelder herauskristallisieren, das Thema der Kompilation insgesamt auch aufgrund einiger schwer miteinander zu vereinbarender Schlussfolgerungen zuweilen sehr stark aufgefächert wirkt. Dies mag darin begründet liegen, dass die zweite Phase seiner Erforschung aufgrund der Ambiguität des Gegenstandes sehr divergente Ergebnisse produzierte und hier nachwirkt. Dem Anliegen der Autoren entsprechend trägt jedoch gerade dies mit dazu bei, dass die Argumentationen in diesem gelungenen Sammelband vielseitig und über die Forschung zu den New Spiritualities hinaus anschlussfähig sind. 